This item was submitted to Loughborough's Institutional Repository (https://dspace.lboro.ac.uk/) by the author and is made available under the following Creative Commons Licence conditions.

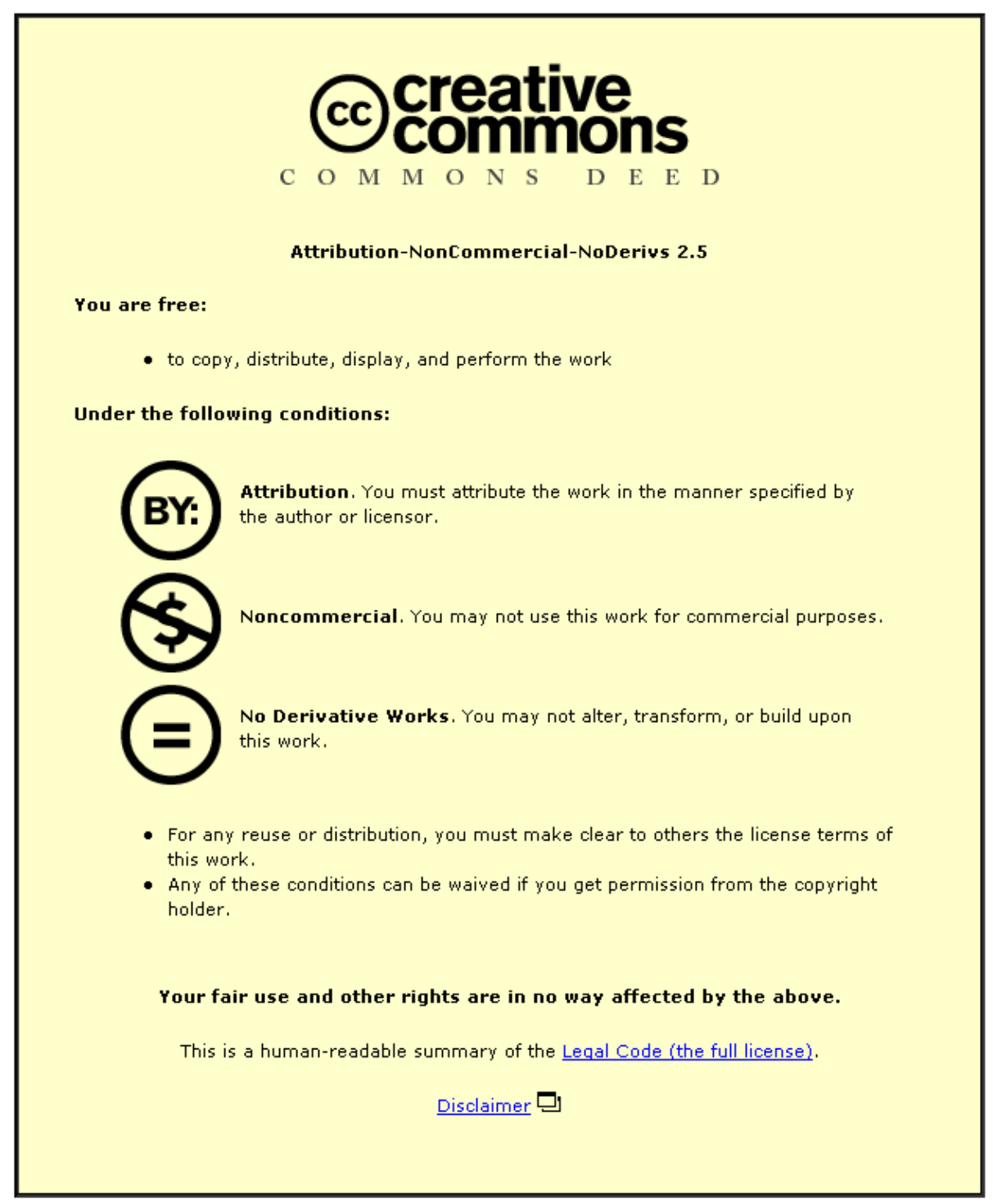

For the full text of this licence, please go to: http://creativecommons.org/licenses/by-nc-nd/2.5/ 


\title{
TRANSPORT PROCESSES DURING ELECTROWASHING OF FILTER CAKES
}

\author{
R. Kilchherr ${ }^{1}$, M.A. Koenders ${ }^{1}$, R.J. Wakeman ${ }^{2}$ and E.S. Tarleton ${ }^{2}$ \\ ${ }^{1}$ Dept. of Mathematics, Kingston University, Penrhyn Road, Kingston on Thames, Surrey, UK. \\ ${ }^{2}$ Advanced Separation Technologies Group, Dept. Chemical Engineering, Loughborough \\ University, Loughborough, Leics., UK.
}

\begin{abstract}
Applications of electric fields during washing of filter cakes increases the removal rate of ions from the cake mother liquor, and under appropriate conditions the field also increases the wash flow rate by electroosmosis. Experimental data that show the key effects of the fields on the rates of ion mass transfer are presented: with the downstream electrode acting as a cathode, cation removal rates are increased whilst the removal rate of the anions is decreased. The concentration profile of the cations with washing time shows an increase in concentration to a value above that of the mother liquor, before it decreases due to displacement by the fresh wash liquor. A model is formulated that describes the advection, dispersion, ion migration and electroosmosis transport processes in the cake. Numerical solution of the model gives cation concentration profiles at the exit of the cake that are in qualitative agreement with the experimental observations.

Experimentally measured wash liquor flow rates tend to be lower than what traditional colloid science principles predict by a factor of 5 to 10: reasons for this difference, supported by experimental work from other researchers, are discussed.
\end{abstract}

\section{KEYWORDS}

Electroosmosis; Modelling; Porous media; Transport processes; Filter cake washing

\section{INTRODUCTION}

Filter cake washing assisted by electrical fields is a new area of technology that has received only limited attention by researchers ${ }^{1-3}$. Although only slight increases in the kinetics of washing have been reported ${ }^{2}$, the situation can be quite different when the removal of specific ions is measured ${ }^{3}$. Electro-osmotic flow of ions in porous systems under the action of an electrical field is of practical interest for soil stabilisation ${ }^{4}$, contaminated soil remediation ${ }^{5-9}$ the dewatering of tailings ponds ${ }^{10,11}$ and the dewatering of sewage sludge ${ }^{12-18}$.

Fundamental theory to describe the observed phenomena in the context of soil remediation is available ${ }^{19,20}$. In the modelling employed in these papers it is tacitly assumed that ionic diffusion is entirely due to thermal processes (see for example Lyklema ${ }^{21}$ ). This is valid because the granular aggregate typically consists of sand grains with a mean particle diameter in the order 50-100 $\mu \mathrm{m}$. In the electrowashing experiments, described below, the mean diameter is an order of magnitude smaller. As a consequence physical effects are important that become manifest at pore sizes that are of the same order of magnitude as the double layer thickness. Similarly, in electroosmosis through sintered glass ${ }^{22}$ with grain sizes similar to sands.

Experiments and theory in the related area of sludge dewatering by electro-osmotic means make use of the same concepts ${ }^{23}$. Earlier, the same authors ${ }^{24}$ also studied electroosmosis combined with pressure-induced flow through a packed bed of particles of mean diameter of approximately $10 \mu \mathrm{m}$. While this value is still too large to see effects associated with the double layer and pore diameter being of the same order of magnitude, many physical concepts in that paper are pertinent, especially the recognition that a packed particle bed is intrinsically heterogeneous. They 
also show that the chemical composition of the particles in the packed bed remains unaltered. This implies that effects due to chemical reactions that could conceivably have taken place at certain - possibly critical - porosities, do not occur.

In this paper, experiments are reported in which the mean particle size is about $0.3 \mu \mathrm{m}$. The double layer thickness is varied by manipulating the ionic strength and effects come to the fore that are associated with the two length scales coming together. First the experiments are described, as well as the salient experimental results. Then the experiments are modelled, using a simple phenomenological, linear model that brings out the main features of the electro-washing process. In a subsequent section the various length scales are investigated and the key physical processes that take place are discussed, thus underpinning the choices of the values made for the phenomenological parameters.

\section{EXPERIMENTS: APPARATUS AND MATERIALS}

An automated experimental apparatus that facilitated downward constant pressure filtration and displacement cake washing is shown schematically in Figure 1. The arrangement, made from stainless steel, comprised suspension and wash liquor feed reservoirs and a means for delivering compressed air to the filter at a controlled rate to maintain a constant pressure. Liquid from downstream of the filter test cell (area $120 \mathrm{~cm}^{2}$ ) was either directed to a PC interfaced electronic balance or, during washing, collected in 1 of 20 sample bottles mounted on a rotary indexing table. A PC was used to record the mass of liquids discharged from the filter and the filtration/washing pressures and also to control the air pressure on the feed side of the filter cell. Iridium coated titanium mesh was chosen as a reasonably inert material for both the upper and lower electrodes. The upper electrode was positioned such that it touched the top of the cake surface when formation was complete. The lower electrode was just below the underside of the porous metal support on which the $0.2 \mu \mathrm{m}$ rated Gelman Versapor filter medium rested. In all experiments the electrode spacing was maintained at $13 \mathrm{~mm}$ to accommodate the thickness of the cake, membrane and support. A DC power supply (SM 35-45) manufactured by Delta Elektronika BV was connected to the electrodes inside the filter cell and operated in the constant voltage mode. The voltage was applied such that the anode was above the filter medium/cake.

An $8.6 \% \mathrm{v} / \mathrm{v}$ suspension of titania $\left(\mathrm{TiO}_{2}\right.$ rutile: density $4260 \mathrm{~kg} \mathrm{~m}^{-3}$, mean particle size $\left.\sim 0.3 \mu \mathrm{m}\right)$ in a solution of $0.001 \mathrm{M}$ or $0.01 \mathrm{M} \mathrm{NaNO}_{3}$ was initially prepared by adding a known amount of the dry powder to an aqueous $\mathrm{NaNO}_{3}$ solution. The resultant mixture was homogenised using an UltraTurrax T25 homogeniser (Janke \& Kunkel, IKA Labortechnik) with a $25 \mathrm{~mm}$ dispersing tool at 8000 rpm for approximately 20 minutes. The $\mathrm{pH}$ of all feed suspensions was adjusted to the required value with either nitric acid or sodium hydroxide. A stock salt solution was made by adding $\mathrm{NaNO}_{3}$ into deionised (DI) water supplied from a Millipore MilliRX20 water purification system; the $\mathrm{Na}^{+}$and $\mathrm{NO}_{3}{ }^{-}$were used as the tracer ions to track the progress of the displacement washing phase. Zetapotentials for rutile were measured with a Malvern Instruments ZetaSizer and the variation of zetapotential with $\mathrm{pH}$ is shown in Figure 2. Measurements were carried out at two different ionic strengths over the $\mathrm{pH}$ range 3.5 to 9 , where the zeta-potential decreased with increasing $\mathrm{pH}$. For a $0.001 \mathrm{M}$ solution the IEP occurred at $\mathrm{pH} 5.2$ whereas for $0.01 \mathrm{M}$ solution a pH of 5.8 corresponded to the IEP.

Prior to each experiment the pipework throughout the apparatus was rinsed with DI water to ensure that it was free of any particle/solute residue from earlier experiments. Suspension at the required $\mathrm{pH}$ and ionic strength and $\mathrm{DI}$ water were added into the suspension vessel and washing liquid vessel respectively. A filter cake of the required $10 \mathrm{~mm}$ thickness was formed in the conventional manner by constant pressure filtration in the absence of an electric field. Cake formation was followed by a displacement washing phase using DI water either with or without the application of an electric field. In a typical sequence of experiments the applied voltage was varied over the range 0 to $30 \mathrm{~V}$. During the course of cake washing, both the hydraulic washing pressure 
and the voltage remained constant and up to 20 wash liquor samples were collected and subsequently analysed for ion concentration. The cations were measured using atomic absorption spectroscopy (Varian SpectrAA-200) and the anions using liquid chromatography (Dionex Chromatography). Ions initially present on the surface of the rutile particles were identified using X-ray photoelectron spectroscopy (XPS). The moisture content and porosity of a filter cake were determined using a gravimetric analysis either before or after the washing phase depending on the nature of an experiment.

\section{EXPERIMENTAL RESULTS}

The experiments were designed to observe the effects of the electric field, ionic strength, $\mathrm{pH}$ (zeta potential) and electrode polarity on cake washing in terms of wash liquor flow rates and ion concentrations. The data are reported as washing curves, a plot of normalised instantaneous solute concentration $\left(\mathrm{C} / \mathrm{C}_{0}\right)$ in the wash effluent versus wash time $(t)$.

Figures 3 and 4 show how cation $\left(\mathrm{Na}^{+}\right)$and anion $\left(\mathrm{NO}_{3}{ }^{-}\right)$concentrations in the wash liquor varied with wash time and applied voltage. In these experiments the filtration pressure $\left(\Delta p_{f}\right)$ and washing pressure $\left(\Delta p_{w}\right)$ were $400 \mathrm{kPa}$ and the initial suspensions were prepared in $10^{-3} \mathrm{M} \mathrm{NaNO}_{3}$ and adjusted to $\mathrm{pH}$ 7.8. For these conditions, the suspensions exhibited a zeta potential of $-47 \mathrm{mV}$. During washing without an electric field, the instantaneous $\mathrm{Na}^{+}$ion concentration in the wash liquor decreased in the initial period up to $\sim 600 \mathrm{~s}$ and then reduced more rapidly with a tendency toward a limiting value. When an electric field was applied the normalised concentration of $\mathrm{Na}^{+}$ions initially increased with time and reached the maximum values of 1.02 (at $10 \mathrm{~V}), 1.18($ at $20 \mathrm{~V}$ ) and 1.24 (at $30 \mathrm{~V}$ ), implying an accelerated removal of sodium ions from the cake with increasing electric voltage. A mass balance on the sodium ions washed from the cake suggested that some removal of $\mathrm{Na}^{+}$ions from the particle surfaces may also have occurred; additional reslurry washing experiments without an electric field showed that there were $\mathrm{Na}^{+}$ions on the particle surfaces that were removable by washing. In general, an electric field improved cake washing most effectively during the initial stages of washing and when the electric field strength was greater.

At longer washing times the $\mathrm{Na}^{+}$concentration is higher when an electric field is applied than is the case when there is no applied potential. The implication from the 'tails' of the curves on Figure 3 is that there is a slow mechanism removing ions from the filter cake and that more ions are removed when an electric field is applied (this is reinforced when the area under the curves is calculated, showing more $\mathrm{Na}^{+}$removal at higher applied voltages).

Regarding the $\mathrm{NO}_{3}{ }^{-}$anions, Figure 4 shows how the washing curve changed as the applied voltage was raised. The number of anions removed in the wash liquid decreased with an increasing electric field. When the downstream electrode is the cathode, the anions within the cake are subjected to an additional upward velocity. The direction of motion of the anions opposes both the electro-osmotic flow and the flow induced by the hydraulic pressure gradient. The force causing counter-flow of the anions becomes more dominant as the applied voltage is increased.

A sequence of washing experiments with $10^{-2} \mathrm{M} \mathrm{NaNO}_{3}$ were also performed; all other conditions remained the same. Results showed the same general trends as those recorded in Figures 3 and 4. A slightly increased maximum dimensionless $\mathrm{Na}^{+}$concentration was noted when an electric field was applied (e.g. at $30 \mathrm{~V},\left(C / C_{0}\right)_{\max }$ was 1.29 compared with 1.24 for the $10^{-3} \mathrm{M}$ case). For a raised $\mathrm{Na}^{+}$ion concentration in the wash liquor there was generally a reduction in the $\mathrm{NO}_{3}{ }^{-}$ion concentration indicating a reduced washing performance with respect to anion removal.

Wash liquor flow rates were generally higher at $10^{-2} \mathrm{M} \mathrm{NaNO}_{3}$. The cake porosities were almost identical at each molar concentration, suggesting that there was an increased contribution from electroosmosis. As more salt is added to the initial suspension the double layer shrinks, tending to reduce the zeta potential of the rutile particles (Figure 2). At the higher ionic strength a larger 
current and power were consumed, particularly toward the beginning of washing were the ion concentration was at its highest (a maximum power $\sim 30 \mathrm{~W}$ was observed for $\Delta V=30 \mathrm{~V}$ ). In the cases where an electric field was applied, the current decayed to a low value within a short period and this decay was more rapid at raised field strengths.

To assess the impact of zeta potential on electrowashing, filter cakes were also formed from suspensions close to their IEP $\left(10^{-2} \mathrm{M} \mathrm{NaNO}_{3} ; \mathrm{pH} 5.2\right)$ and subsequently washed both with and without applied electric fields. Due to higher filter cake porosities it was necessary to reduce both filtration and washing pressures to $100 \mathrm{kPa}$ to facilitate the experimental measurements. The results showed that both anions and cations behave in a generally similar manner to that noted for electrowashing at $\mathrm{pH} 7.8$, although lower flow rates were recorded.

The variation of cake porosity with wash time was determined by performing two series of otherwise identical experiments where either no field was present or a $20 \mathrm{~V}$ potential was applied between the electrodes. Within each series, an experiment was stopped at a set time and cake samples taken to gravimetrically determine a porosity value corresponding to a given position on the wash curve. For the case of no electric field the largest change in cake porosity was noted during the first few minutes of washing after which there was essentially no further change. However, with an applied voltage of $20 \mathrm{~V}$ the average cake porosity was seen to fall from an initial value of 0.57 to a near constant value of 0.47 after a wash time of $\sim 1000 \mathrm{~s}$. At all times following the start of washing the cake porosity with no electric field exceeded that measured at an identical time with an applied field of $20 \mathrm{~V}$.

Figures 5 and 6 show the effect of increasing ionic strength in the cake mother liquor. In these experiments the filtration pressure $\left(\Delta p_{f}\right)$ and washing pressure $\left(\Delta p_{w}\right)$ were $100 \mathrm{kPa}$. The microprocesses involved during electrowashing vary with the ionic strength due to the effect of the latter on the state of dispersion of the particle prior to their filtration, which reflects on the porosity $(\varepsilon)$ of the cake at the start of washing. For example, whereas at $3 \mathrm{M} \varepsilon$ is 0.626 at the end of cake formation and during washing reduces to 0.527 , at $1 \mathrm{M}$ the reduction of $\varepsilon$ is from 0.575 to 0.512 . This lowering of porosity causes a wash liquor flow rate reduction, which may more than offset the wash liquor flow rate increase due to electroosmosis. Nonetheless, the peaks in the washing curves are evident in lower molar concentration feeds, but disappear as the molarity is increased.

\section{MODELLING CONCEPTS}

A co-ordinate frame is chosen such that the electric field is applied in the $x$-direction. Clean water is introduced at $x=0$. The ion concentration measurement takes place at $x=L$. The modelling is done by assuming that there are two pools of ions: those trapped in the pores - denoted by a superscript $(p)$ - and the ones that move more or less freely with either the main fluid flow or the electrically generated ionic current; the latter are distinguished by a superscript (f). It is assumed that the ions in the ( $f$ ) pool effectively move in the $x$-direction. The ions of pool $(p)$ may diffuse into the free flow and to do so they must acquire a motion in a direction that is normal to the $x$-axis. This motion may be highly complicated and for simplicity it is summarised here as 'motion in the $y$ direction'. A similar concept has been developed previously to model diffusional extraction from hydrodynamically stagnant regions in porous media ${ }^{25}$; the concept and notation are summarised in Figure 7.

All ions may come in any number of species. The simplest model involves two species, positive and negative ones. So, there are four ion concentrations to be considered: $C^{(1 f)}, C^{(2 f)}, C^{(1 p)}, C^{(2 p)}$. These are functions of the position $x$ and the time $t$. 
The velocities of the ions relative to the mean fluid velocity in the $x$-direction are: $v^{(1 f)}, v^{(2 f)}, v^{(1 p)}$, $v^{(2 p)}$. These velocity vectors may have two components; the species labelled $(p)$ have a zero $x$ component.

It is convenient to introduce the mass flux vector for each species of ion $\boldsymbol{J}^{(i)}$. The equations of continuity take the following form:

$$
\begin{aligned}
& \frac{\partial C^{(1 f)}}{\partial t}+\frac{\partial J_{x}^{(1)}}{\partial x}+\frac{J_{y}^{(1)}}{a}=0 \\
& \frac{\partial C^{(2 f)}}{\partial t}+\frac{\partial J_{x}^{(2)}}{\partial x}+\frac{J_{y}^{(2)}}{a}=0 \\
& \frac{\partial C^{(1 p)}}{\partial t}-\frac{J_{y}^{(1)}}{a}=0 \\
& \frac{\partial C^{(2 p)}}{\partial t}-\frac{J_{y}^{(2)}}{a}=0
\end{aligned}
$$

where $a$ is an internal length scale (of the order of magnitude of the mean radius of the particle forming the bed), which is introduced for dimensional reasons.

Constitutive equations are now sought to characterise the physics of ionic migration. The velocity in the $x$-direction of the freely moving ions is ruled by the strength of the $x$-component of the local electric field $E$, thus

$$
\begin{aligned}
& J_{x}^{(1)}=A^{(1)} C^{(1 f)} E \\
& J_{x}^{(2)}=A^{(2)} C^{(2 f)} E
\end{aligned}
$$

Here, the coefficients $A^{(\bullet)}$ may be positive and negative, depending on the sign of the charge of the species. Its magnitude depends on the valence and process susceptibility. More details of the physics that is represented in $A^{(\bullet)}$ is given in the Discussion section. Essentially, $A^{(\bullet)} E$ represents a mean velocity and the location of the peak in the output of some of the experiments corresponds to the time that the ions have travelled from one end to the other end of the apparatus. Calling this distance $L$ and letting the species of ion that displays the peak be the (1)-type, then the time at which the peak occurs is

$$
T_{p}=\frac{L}{A^{(1)} E}
$$

Further constitutive refinements may be made to the transport velocity of the freely moving ions, especially diffusive effects should be added. These are proportional to $\partial C^{(f i)} / \partial x$; while they do not lead to non-linearity, such terms are most easily studied in a numerical context and in this first order exploration they are omitted; in the next section more details of diffusion effects are considered.

Now a constitutive form for the $y$-components of the flux is considered. A linear form is put forward here, suggesting that the efficacy with which ions are dislodged from the closed pores into the freeflowing regions is related to the concentration of the various species in the vicinity. Thus, eight constitutive coefficients are introduced as follows: 


$$
\begin{aligned}
& J_{y}^{(1)}=F^{(11)} C^{(1 f)}+F^{(12)} C^{(2 f)}+P^{(11)} C^{(1 p)}+P^{(12)} C^{(2 p)} \\
& J_{y}^{(2)}=F^{(21)} C^{(1 f)}+F^{(22)} C^{(2 f)}+P^{(21)} C^{(1 p)}+P^{(22)} C^{(2 p)}
\end{aligned}
$$

The coefficients may be positive or negative. The character of the process is depends to a large extent on these coefficients. Some preliminary observations may be made. The coefficients $P^{(11)}$ and $P^{(22)}$ are never zero: the out-flux of ions from the pores must be proportional to the available concentration. Similarly, $F^{(11)}$ and $F^{(22)}$ are never zero: the influx of ions into the pores must be proportional to the available concentration in the free flowing pool. It is also easily verified that $P^{(11)}$ and $P^{(22)}$ are both negative and that $F^{(11)}$ and $F^{(22)}$ are both positive. They need not be exact opposites as the probability of an ion migrating into the pore is most likely not equal to the probability of one escaping from it. As to the cross terms, it is likely that it is attractive for a positive ion to migrate from a pore into a region of plentiful negatives. On that basis the cross terms may have an influence and it is expected that $F^{(12)}$ and $F^{(21)}$ are both negative, while $P^{(12)}$ and $P^{(21)}$ are both positive. The cross terms are small when the solutions are dilute. The coefficients $P^{(\cdot \bullet)}$ and $F^{(\bullet \bullet)}$ may also be influenced by any chemical reactions (i.e. adsorption and precipitation) taking place between the $(f)$ ions and the granular medium.

The linear forms put forward here are a first order model; especially for not so dilute systems nonlinear effects must be expected to play a role. Numerical simulation could shed more light on higher order effects, though for a realistic simulation more constitutive parameters are needed, which requires a better insight in the physics of the process than is currently available.

The differential equations that rule the process are obtained by inserting the constitutive equations, equations (8) and (9), into the equations of continuity, equations (1) to (4):

$$
\begin{aligned}
& \frac{\partial C^{(1 f)}}{\partial t}+A^{(1)} E \frac{\partial C^{(1 f)}}{\partial x}+\frac{F^{(11)} C^{(1 f)}+F^{(12)} C^{(2 f)}+P^{(11)} C^{(1 p)}+P^{(12)} C^{(2 p)}}{a}=0 \\
& \frac{\partial C^{(2 f)}}{\partial t}+A^{(2)} E \frac{\partial C^{(2 f)}}{\partial x}+\frac{F^{(21)} C^{(1 f)}+F^{(22)} C^{(2 f)}+P^{(21)} C^{(1 p)}+P^{(22)} C^{(2 p)}}{a}=0 \\
& \frac{\partial C^{(1 p)}}{\partial t}-\frac{F^{(11)} C^{(1 f)}+F^{(12)} C^{(2 f)}+P^{(11)} C^{(1 p)}+P^{(12)} C^{(2 p)}}{a}=0 \\
& \frac{\partial C^{(2 p)}}{\partial t}-\frac{F^{(21)} C^{(1 f)}+F^{(22)} C^{(2 f)}+P^{(21)} C^{(1 p)}+P^{(22)} C^{(2 p)}}{a}=0
\end{aligned}
$$

These equations must be supplemented with boundary conditions and initial conditions. The latter are chosen to be constant in position and the same for all species and types:

$$
C^{(1 f)}(x, 0)=C^{(2 f)}(x, 0)=C^{(1 p)}(x, 0)=C^{(2 p)}(x, 0)=C_{0}
$$

The boundary conditions are as follows

$$
C^{(1 f)}(0, t)=0
$$

That is, fresh water is flushed in at one end of the apparatus. Furthermore, the whole filter cake must stay neutral. Adding the equations of continuity with the correct signs for the charges (assuming the same valency for all ions) gives: 
$\frac{\partial C^{(1 f)}}{\partial t}-\frac{\partial C^{(2 f)}}{\partial t}+\frac{\partial C^{(1 p)}}{\partial t}-\frac{\partial C^{(2 p)}}{\partial t}+\frac{\partial J_{x}^{(1)}}{\partial x}-\frac{\partial J_{x}^{(2)}}{\partial x}=0$

Integrating over the apparatus dimension $L$ yields:

$\frac{\partial}{\partial t} \int_{0}^{L}\left(C^{(1 f)}-C^{(2 f)}+C^{(1 p)}-C^{(2 p)}\right) d x+J_{x}^{(1)}(L, t)-J_{x}^{(1)}(0, t)-J_{x}^{(2)}(L, t)+J_{x}^{(2)}(0, t)=0$

Making use of the fact that the total charge vanishes and inserting the boundary condition at $x=0$ results in

$J_{x}^{(1)}(L, t)-J_{x}^{(2)}(L, t)+J_{x}^{(2)}(0, t)=0$

The solution of equations (10) to (18) is obtained numerically, with the method summarised in Appendix A. This approach requires the coefficients to be known and these are obtained by solving the problem for short times and comparing this solution with experiments. The initial stage of the ion concentration in the apparatus at location $x=L$ (the measurement point) is obtained using Laplace transforms: Appendix $\mathrm{B}$ gives the details. The result up to second order in $t$ is obtained, assuming that the cross terms $F^{(12)}, P^{(21)}, F^{(21)}$ and $P^{(12)}$ are negligible:

$C^{(1 f)}(L, t)=C_{0}-\frac{C_{0}\left(F^{(11)}+P^{(11)}\right)}{a} t+\frac{C_{0}\left(F^{(11)}+P^{(11)}\right)\left(F^{(11)}-P^{(11)}\right)}{a^{2}} t^{2}+\ldots$

The coefficient of the second term in the series in $t$ on the right hand side is the derivative at $t=0$. The output as a function of time increases when $F^{(11)}+P^{(11)}<0$. It is recalled that $F^{(11)}>0$ and $P^{(11)}<0$. Behaviour that leads to a peak is therefore obtained when the probability of ions being released into the free flow from the pores is greater than vice versa.

In order to obtain information for the whole washing curve and not merely the short time approximation, numerical analysis has been used (see Appendix $B$ ). The numerics require input parameters. First an estimate for the velocity of the ions is obtained from the location of the peak. In Figure 8 the data for a $\mathrm{pH}$ value equal to 7.8 is explored. The velocity is determined and should be of the form $v=v_{0}+A^{(1)} E$. The reason is that both the electric field and the fluid pressure difference give the ions a velocity. Figure 8 shows reasonable agreement with this hypothesis.

Below the physics of the velocity will be debated; for the moment the velocity is introduced as a phenomenological parameter. The parameters $P^{(11)}$ and $F^{(11)}$ are varied to give the best correspondence with the experimental outcome (again at $\mathrm{pH} 7.8$ ) and in Figure 9 the calculated curves are shown. The theoretical effect that gives rise to the presence or absence of the peak is recovered in the numerical approach.

In certain cases a curve such as $C$ in Figure 9 displays a bending point. The location of this point may be obtained from the short-time approximation and the following is found:

$$
t_{\text {bend }}=\frac{a}{F^{(11)}-P^{(11)}}
$$

Normally, the value that corresponds to this formula is in the order of hundreds of seconds (see Figures 3 and 5); this is another indication that the order of magnitude chosen for $P^{(11)}$ and $F^{(11)}$ is in the right order of magnitude. This does not mean to say that $P^{(11)}$ and $F^{(11)}$ are constants, 
independent of the applied electric field. To illustrate this the washing curves for two applied velocities are shown at the same values for $P^{(11)}$ and $F^{(11)}$, Figure 10 . It is observed that the slower washing curve shows a higher peak, while the experiments show the opposite effect, implying that the coefficients depend on the applied electric field.

\section{DISCUSSION OF PHYSICAL CONCEPTS}

The pools of ions that are more or less static are assumed to be situated in regions that are slightly more densely packed than the average packing. The free flow will take place in regions that are more loosely packed. Packing variations in granular assemblies are normal for a heterodisperse aggregate. The extent of the heterogeneity has been demonstrated by Koenders and Williams ${ }^{26}$, who, in order to predict correct values for the overall (continuum) permeability, show that packing density variation with a correlation length of some three mean diameter sizes must be accounted for.

The consequence of a packing density variation is local fluctuations in the dielectric constant. This occurs because the fluid and solids have different dielectric constants and therefore a variation in the porosity will lead to a commensurate variation in the dielectric constant. A simple calculation of a spherical inclusion of a region with a higher dielectric constant in an average medium is carried out $^{27}$. The resulting equi-potential lines are shown in Figure 10.

The streamlines, which are normal to the equi-potential lines, are bent in such a way that the inclusion attracts them. The sign convention of the ion flux $J_{y}^{(1)}$ is chosen such that a positive value depletes the ions in the free flow and therefore $F^{(11)}$ is positive, associating it with the motion normal to the free flow into the inclusion on the up-stream side. The quantity $P^{(11)}$ is associated with ions coming out of the inclusion on the downstream side and is negative (it supplies ions to the (f) pool.

The experiments in which a peak in the ion concentration is found are described by taking $\left|P^{(11)}\right|>\left|F^{(11)}\right|$ at small ionic strength and $\left|P^{(11)}\right| \approx<\left|F^{(11)}\right|$ at high ionic strength. It is also seen (through the exercise of Figure 11) that $F^{(11)}$ and $P^{(11)}$ are themselves dependent on the applied electric field. The fact that a change in the $\zeta$-potential down to the isoelectric point makes little difference to the outcome of the experiments (see Figure 9) implies that the parameters $F^{(11)}$ and $P^{(11)}$ are largely independent of the electroosmotic effect, but instead are dominated by ionic migrational features. Using some of the experimental data, this will be further explored below.

Length scales are important for the problem. The double layer thickness $\kappa^{-1}$ depends on the ionic strength $^{21}$. At $0.001 \mathrm{M}$ the double layer thickness is some $10 \mathrm{~nm}$; this reduces (with the root of the ionic strength) to some $0.25 \mathrm{~nm}$ at $3.0 \mathrm{M}$. The mean diameter of the cake particles $\left(D_{50}\right)$ is in the order of $340 \mathrm{~nm}$. Depending on the porosity of the cake a typical effective pore diameter exists. To estimate the order of magnitude of this parameter two approaches may be used. In the first, geotechnical filter stability rules are invoked; these give the mean diameter of a particle that can just pass through a packed bed. According to Terzaghi ${ }^{28}$ the value of the critical diameter is $D_{15} / 5$. For the particles used in this work the uniformity of the size distribution $\left(D_{60} / D_{10}\right)$ is 4.2 , and $D_{15} \approx$ $140 \mathrm{~nm}$, leaving a mean pore size of the order of $28 \mathrm{~nm}$. In the other approach the gap width between the particles is estimated ${ }^{29}$. This estimate depends more explicitly on the solidosity and yields values between $10 \mathrm{~nm}$ to $20 \mathrm{~nm}$ in the solidosity range $0.4<\varphi<0.6$ (the range measured in the experiments was $0.43<\varphi<0.53$ ). Naturally, these estimates are averages; in a packed bed an enormous range of values is encountered. 
A charged ion in solution behaves as an entity with an effective diameter of the order of $2 \kappa^{-1}$. Thus it is seen that the pore diameter and the diameter of the ionic entity are of the same order of magnitude at the ionic strength of $0.001 \mathrm{M}$, while below $0.1 \mathrm{M}$ the ionic entity diameter is well below the mean pore size. The natural fluctuations in packing density together with the fact that the streamlines must go through the denser regions has implications for the energy of the ions. When the ionic entity is much smaller than the diameter of the pore, the ion has a greater mean free path (lower potential energy), than when it is trapped in a pore with a diameter that is of the same order of magnitude. It is therefore energetically attractive for ions to escape from the denser regions at large double layer thicknesses. For thin double layers the energetic gain/loss of moving into and out of the dense inclusion is negligible.

These considerations are now be phrased in terms of a diffusion theory. From the outset it is noted that this type of diffusion takes place on a meso-scale with a length scale of a few grain diameters. Thermal diffusion, by contrast, takes place on the molecular scale. Equation (8) (which acts as a constitutive equation for the flux into and out of the free flow) neglecting cross terms reads:

$J_{y}^{(1)}=F^{(11)} C^{(1 f)}+P^{(11)} C^{(1 p)}$

The mean ion density in the vicinity of the inclusion is called $\langle C\rangle$; therefore $C^{(1 f)}=\langle C\rangle+a \partial C / \partial y$ and $C^{(1 p)}=\langle C\rangle-a \partial C / \partial y$. Expanding the coefficients in equation (21) in a similar way leaves

$J_{y}^{(1)}=\left(F^{(11)}+P^{(11)}\right)\langle C\rangle+\left(F^{(11)}-P^{(11)}\right) a \frac{\partial C}{\partial y}$

This constitutive equation describes the average flux in the whole region in the vicinity of the inclusion. The second term on the right hand side equation (22) represents a diffusion term. The diffusion coefficient is, in the usual way, proportional to the product of the mean fluctuation speed $\langle v\rangle$ and the mean free path $\lambda$. The former follow from the fluctuations in the electric field in the vicinity of the inclusion and are themselves proportional to the mean applied electric field. The local pore geometry also plays a role. The mean free path of the ionic entities depends on the double layer thickness as outlined above.

Noting the signs of $F^{(11)}$ and $P^{(11)}$ it is observed that $\left(F^{(11)}-P^{(11)}\right)$ represents an average and that $\left(F^{(11)}+P^{(11)}\right)$ a first order variation. Therefore, the latter must be proportional to a variation in $\langle v\rangle \lambda$; hence

$F^{(11)}+P^{(11)} \propto \frac{\partial(\langle v\rangle \lambda)}{\partial y} a$

As both the mean fluctuation speed and the mean free path are smaller inside the inclusion when the ionic entities are more impeded in their movements, it follows that $\left(F^{(11)}+P^{(11)}\right)<0$ for thick double layers and that $\left(F^{(11)}+P^{(11)}\right) \rightarrow 0$ when $\langle v\rangle \lambda$ approaches its thermal value for the case of the thin double layer relative to the pore diameter.

The order of magnitude of the velocity is now discussed. Following Yu and Neretniks ${ }^{20}$ three factors are involved. These are: (1) the ionic migrational flux, (2) the electro-osmotic flux, and (3) the applied fluid pressure difference related mean flow. Other mechanisms play a negligible role. Best estimates are obtained as follows: 
(1) Following $Y u$ and Neretnieks ${ }^{20}$ The ionic migration flux $J^{m}$ is

$J^{m}=\frac{z}{|z|} \frac{D|z| F}{R T} C E$

where $z$ is the ionic valency, $D$ the diffusion coefficient, $F$ the Faraday's constant, $R$ the gas constant, $C$ the concentration and $T$ the absolute temperature. All the physical constant are readily known; for $\mathrm{Na}$ the valency equals unity and the diffusion coefficient is $D=1.3 \times 10^{-9} \mathrm{~m}^{2} \mathrm{~s}^{-1}$. The electric field follows simply from the applied potential divided by the cell height $L(L=0.01 \mathrm{~m})$. The flux is $J^{m}=v_{(1)} C$ and it is seen that an estimate for $v_{(1)}$ is easily obtained.

(2) The electro-osmotic flux $J^{e}$ follows from Probstein and Hicks ${ }^{19}$, Hunter ${ }^{30}$

$J^{e}=-\frac{\varepsilon \zeta E}{\mu} C$

where $\varepsilon$ is the permittivity of the fluid $\left(\varepsilon=\varepsilon_{\mathrm{r}} \varepsilon_{0}\right), \zeta$ the zeta potential and $\mu$ the viscosity of the fluid. The estimate for the velocity is obtained by assuming a relative permittivity $\varepsilon_{r}=80$, a zeta potential $-\zeta=48$ or $1 \mathrm{mV}($ at $\mathrm{pH} 7.8$ or $5.2,0.001 \mathrm{M}$ ) and $-\zeta=42 \mathrm{mV}$ (at pH 7.8, $0.01 \mathrm{M}$ ), and a viscosity $\mu$ $=0.001 \mathrm{~Pa} \mathrm{~s}$.

(3) The third flux is estimated from the experimental data when no electric field is applied.

Using the best estimates, the flux contributions are summarised in Table 1 for some of the experiments that can be interpreted in this way.

Note that compared to the experiments, the ratio of velocities is reproduced more or less correctly, but the absolute value of the theoretical calculations is roughly a factor of 5 to 10 too high. There are two reasons why this is so. The first is that in the evaluation of the field-induced velocities a straight path is assumed. In reality the path is tortuous. For regular assemblies a factor of $2^{0.5}$ is introduced. For irregularly packed aggregates the factor is higher. Qualitative evidence for this is found in a study by Weber and Kimmich ${ }^{31}$, using NMR velocity mapping to image the flow of ions through a porous medium, revealing tortuous flow paths with a tortuosity that is much greater than $2^{0.5}$; values up to 5 are obtained there. The reason that only qualitative evidence can be derived from this is that these measurements are done in two dimensions on a pixel medium and not on a three dimensional granular medium. A further reason for the deviation of the theoretically predicted velocity from the experimental value is related to the problem discussed at the beginning of this section: the fact that the double layer extends the radius of the ionic entity to a value that approximates the pore size. In other words, in a porous medium the thermal diffusion coefficient is not valid.

\section{CONCLUSIONS}

In this paper the effects of imposing an electric field on an otherwise conventional displacement washing process are analysed through a phenomenological model. The model parameters were obtained through modelling experimental data.

Applications of electric fields during washing of filter cakes increases the removal rate of ions from the cake mother liquor, and under appropriate conditions the field also increases the wash flow rate by electroosmosis. From the data it can be concluded that the kinetics of ion removal from the cake mother liquor can be enhanced substantially by an electric field: with the downstream electrode acting as a cathode, cation removal rates are increased whilst the removal rate of the 
anions is decreased. The concentration profile of the cations with washing time shows an increase in concentration to a value above that of the mother liquor, before it decreases due to displacement by the fresh wash liquor. The effectiveness of the applied field is dependent on the ionic concentration in the liquid in the pores of the cake at the start of washing, with a greater apparent mobility of cations being observed at lower solution molarity.

A model is formulated that describes the advection, dispersion, ion migration and electroosmosis transport processes in the cake. Numerical solution of the model gives cation concentration profiles at the exit of the cake that are in qualitative agreement with the experimental observations. Experimentally measured wash liquor flow rates tend to be lower than what traditional colloid science principles predict by a factor of 5 to 10: reasons for this difference, supported by experimental work from other researchers, are attributed to the tortuosity of the pores in the cake and to the fact that the extent of the double layer of the ion extends to the order of magnitude of the pore size..

\section{ACKNOWLEDGEMENTS}

The authors acknowledge receipt of a grant from the Engineering and Physical Sciences Research Council in support of this work.

\section{NOTATION}

$\begin{array}{ll}a & \text { internal length scale }(\mathrm{m}) \\ A^{(\bullet)} & \text { ionic migrational mass flux coefficient } \\ C & \text { concentration of ions }\left(\mathrm{kg} \mathrm{m}^{-3}\right) \\ C_{0} & \text { initial ion concentration }\left(\mathrm{kg} \mathrm{m}^{-3}\right)\end{array}$

$C^{(1 f)}$ concentration of ions of type 1 that move more or less freely with either the main fluid flow or the electrically generated ionic current $\left(\mathrm{kg} \mathrm{m}^{-3}\right)$

$C^{(1 p)}$ concentration of ions of type 1 trapped in the pores $\left(\mathrm{kg} \mathrm{m}^{-3}\right)$

$D_{n} \quad$ diameter of particles such that $\mathrm{n} \%$ of the aggregate (by weight) has size less than $D_{n}(\mathrm{~m})$

E magnitude of the electric field $\left(\mathrm{V} \mathrm{m}^{-1}\right)$

$F \quad$ Faraday's constant $\left(\mathrm{C} \mathrm{mol}^{-1}\right)$

$F^{(\bullet \bullet)} \quad$ coefficient representative of the influx of ions into the pores $\left(\mathrm{m} \mathrm{s}^{-1}\right)$

$\boldsymbol{J}^{(i)}$ mass flux vector, superscript change for the various type of ions $\left(\mathrm{kg} \mathrm{m}^{-2} \mathrm{~s}^{-1}\right)$

$J^{m} \quad$ ionic migration flux $\left(\mathrm{kg} \mathrm{m}^{-2} \mathrm{~s}^{-1}\right)$

$L \quad$ thickness of the filter cake $(\mathrm{m})$

$\Delta p_{f} \quad$ filtration pressure $(\mathrm{Pa})$

$\Delta p_{w} \quad$ washing pressure $(\mathrm{Pa})$

$P^{(\bullet \bullet)} \quad$ coefficient representative of the out-flux of ions from the pores $\left(\mathrm{m} \mathrm{s}^{-1}\right)$

$R \quad$ universal gas constant $\left(\mathrm{J} \mathrm{mol}^{-1} \mathrm{~K}^{-1}\right)$

$T$ absolute temperature $(\mathrm{K})$

$v^{(11)} \quad$ velocity of the ions relative to the mean fluid velocity in the $x$-direction (for 'free' ions of type 1) $\left(\mathrm{m} \mathrm{s}^{-1}\right)$

$\langle v\rangle \quad$ mean fluctuation speed $\left(\mathrm{m} \mathrm{s}^{-1}\right)$

$\lambda \quad$ Length of the mean free path $(\mathrm{m})$

$\mu \quad$ Viscosity of the fluid (Pa s)

$\varphi \quad$ Solidosity

\section{REFERENCES}


1. Larue O., Mouroko-Mitoulou T. and Vorobiev E., 2001, Filtration, cake washing and pressurised electroosmotic dewatering of a highly conductive silica suspension, Trans. Filt. Soc., 1(2), 31-37.

2. Ghirisan A., Weber K. and Stahl W., 2002, The influence of an electrical field on filtration and washing, Trans. Filt. Soc., 2(3), 56-60.

3. Tarleton E.S., Wakeman R.J. and Liang Y., 2003, Electrically enhanced washing of ionic species from fine particle filter cakes, Trans IChemE, 81(A), 201-210.

4. Farmer I.W. and Bell F.G., 1975, Methods of Treatment of Unstable Ground, Butterworths, London.

5. Bruell C.J., Segall B.A. and Walsh M.T., 1992, Electroosmotic removal of gasoline hydrocarbons and TCE from clay, J. Environmental Engineering-ASCE, 118, 68-83.

6. Li Z.M., Yu J.W and Niretnieks I., 1997, Removal of $\mathrm{Cu}(\mathrm{II})$ and $\mathrm{Cr}(\mathrm{III})$ from naturally contaminated loam by electromigration, J. Environ. Sci. HIth., A32, 1293-1308.

7. LeHecho I., Tellier S. and Astruc M., 1998, Industrial site soils contaminated with arsenic or chromium: Evaluation of the electrokinetic method, J. Environmental Technology, 19, 10951102.

8. Kawashi T. and Kubo H., 1999, Model Experimental study on the migration behaviour of heavy metals in electrokinetic remediation process for contaminated soil, Soil Science and Plant Nutrition, 45, 259-268.

9. Kim J. and Lee K., 1999, Effects of electric field directions on surfactant enhanced electrokinetic remediation of diesel contaminated sand column, J. Environ. Sci. Health, A34, 863-877.

10. Lockhart N.C. and Strickland R.E., 1984, Dewatering coal washery ponds by electroosmosis, Powder Technology, 40, 215-221.

11. Chen H., Mujumbar A.S. and Raghavan G.S.V., 1996, Laboratory experiments on electroosmotic dewatering of vegetable sludge and mine tailings, J. Drying Technology, 14, 2435-2445.

12. Kondoh S. and Hiraoka M., 1990, Commercialization of pressurised electroosmotic dehydrator, J. Water Science and Technology, 22, 259-268.

13. Buijs P.J., van Diemen A.J.G. and Stein H.N., 1994, Efficient dewatering of waterworks sludge by electroosmosis, J. Colloids and Surfaces, 85, 29-36.

14. Laursen S., 1993, Electrokinetics in clays and filter cakes activated sludge, Water Science and Technology, 28, 181-188.

15. Laursen S. and Birgerjensen J., 1993, Electroosmosis in filter cakes of activated sludge, Water Research, 27, 777-783.

16. Rabie H.R., Mujumdar A.S. and Weber M.E., 1994, Electroosmotic dewatering of clay suspensions, J. Separations Technology, 4, 38-46. 
17. Barton W.A., Miller S.A. and Veal C.J., 1999, The electrode dewatering of sewage sludges, J. Drying Technology, 17, 497-522.

18. Gingerich I., Neufeld R.D., and Thomas T.A., 1999, Electroosmotically enhanced sludge pressure filtration, Water Environment Research, 71, 267-276.

19. Probstein R.F. and Hicks R.E., 1993, Removal of contaminants from soils by electric fields, Science, 260, 498-503.

20. Yu J. and Neretnieks I., 1996, Modelling of transport and reaction processes in a porous medium in an electrical field, Chem. Eng. Sci., 51(19), 4355-4368.

21. Lyklema J., 1991, Fundamentals of Interface and Colloid Science Vol. I, Academic Press, London.

22. Kobayashi K., Hakoda M., Hosoda Y., Iwata M. and Yukawa H., 1979, Electroosmosis flow through particle beds and electroosmotic pressure distribution, J. Chem. Eng. Japan, 12, 492494.

23. Iwata M., Igami H., and Murase T., 1991, Analysis of electroosmotic dewatering, J. Chem. Eng. Japan, 24(1), 45-50.

24. Yukawa H., Kobayashi K., Yoshida H. and Iwata M., 1979, Studies of electrically enhanced sedimentation, filtration and dewatering processes in Progress in Filtration and Separation Vol. 1, R.J. Wakeman (Ed.), pp.83-111, Elsevier, Amsterdam.

25. Wakeman R.J., 1976, Diffusional extraction from hydrodynamically stagnant regions in porous media, Chem. Eng. J., 11, 39-56.

26. Koenders M.A. and Williams A., 1992, Flow equations of particle/fluid mixtures, Acta Mechanica, 92, 91-116.

27. Jackson J.D., 1962, Classical Electrodynamics, Wiley, New York.

28. Terzaghi K., 1943, Theoretical Soil Mechanics. Wiley, New York.

29. Torquato S., Lu B. and Rubinstein K., 1990, Nearest-neighbour distribution functions in manybody systems, Phys Rev A, 41(4), 2059-2074.

30. Hunter R.J., 2001, Foundations of Colloid Science, Oxford University Press, New York.

31. Weber M. and Kimmich R., 2002, Maps of current density and hydrodynamic flow in porous media: NMR experiments and numerical simulations, Phys. Rev. E, 66, 026306.

32. Carslaw H.S. and Jaeger J.C., 1959, Conduction of Heat in Solids, Clarendon Press, Oxford. 


\section{APPENDIX A: SOLUTION OF EQUATIONS (10)-(18) FOR SHORT TIMES}

To solve equations (10)-(18) a Laplace transform is employed to deal with the temporal part of the equations. The transform is denoted by $a^{\wedge}$ and the Laplace frequency is called $s$. The resulting spatial differential equation set is solve by setting

$$
\begin{aligned}
& \hat{C}^{(1 f)}(x)=\hat{C}_{0}^{(1 f)} e^{\lambda(s) x}+\hat{C}^{(1 f)} \\
& \hat{C}^{(2 f)}(x)=\hat{C}_{0}^{(2 f)} e^{\lambda(s) x}+\hat{C}^{(2 f)} \\
& \hat{C}^{(1 p)}(x)=\hat{C}_{0}^{(1 p)} e^{\lambda(s) x}+\hat{C}^{(1 p)} \\
& \hat{C}^{(2 p)}(x)=\hat{C}_{0}^{(2 p)} e^{\lambda(s) x}+\hat{C}^{(2 p)}
\end{aligned}
$$

To solve the coefficients $\hat{c}^{(\bullet \bullet)}, \hat{C}_{0}^{(\bullet \bullet)}$ and $\lambda(s)$ a symbolic manipulation package DERIVE is employed. The solution to the equations is now obtained assuming that the cross terms $F^{(12)}$, $P^{(21)}, F^{(21)}$ and $P^{(12)}$ are negligible (including these makes the solution rather complex to write down). The solution for $\hat{C}^{(1 f)}(x, s)$ is

$$
\hat{C}^{(1 f)}(x, s)=\frac{C_{0}\left(a s-2 P^{(11)}\right)}{s\left(F^{(11)}-P^{(11)}+a s\right)}\left(1-e^{\lambda(s) x}\right)
$$

where $\lambda(s)=\frac{s\left(F^{(11)}-P^{(11)}+a s\right)}{A^{(1)} E\left(P^{(11)}-a s\right)}$

The value of $A^{(1)} E$ is positive for the ions of species (1). The inverse transform is somewhat complex. The behaviour for small times is instructive, however, and is evaluated at the exit point of the flow $x=L$. The regime $t \rightarrow 0$ corresponds to $s \rightarrow \infty$. Now, $\lambda(s) \rightarrow-s /\left(A^{(1)} E\right)$, so that $\exp (\lambda L) \rightarrow 0$. For $s$ large but not infinite the form $s^{-1} \exp (\lambda(s) L)$ has the inverse transform, which is the step function $u\left(t-L /\left(A^{(1)} E\right)\right)$, indicating that the supply of ions is cut off for times greater than $L I\left(A^{(1)} E\right)$. This point roughly coincides with the peak, if there is one, in the output of the ion concentration as a function of time.

For short times (large values of $s$ ) the solution may be expanded as follows

$$
\hat{C}^{(1 f)}(L, s)=\frac{C_{0}}{s}-\frac{C_{0}\left(F^{(11)}+P^{(11)}\right)}{s^{2} a}+\frac{C_{0}\left(F^{(11)}+P^{(11)}\right)\left(F^{(11)}-P^{(11)}\right)}{s^{3} a^{2}}+\ldots
$$

In the time domain this behaves as

$$
C^{(1 f)}(L, t)=C_{0}-\frac{C_{0}\left(F^{(11)}+P^{(11)}\right)}{a} t+\frac{C_{0}\left(F^{(11)}+P^{(11)}\right)\left(F^{(11)}-P^{(11)}\right)}{a^{2}} t^{2}+\ldots
$$


As the analytical approach obviously has some limitations, the differential equation is solved by a numerical method. Only one type of ion will be taken into account during this simulation. Equation (1) reads

$$
\frac{\partial C^{(1 f)}}{\partial t}+\frac{\partial J_{x}^{(1)}}{\partial x}+\frac{J_{y}^{(1)}}{a}=0
$$

In order to do the numerical analysis each term of equation (B1) the differentiations are replaced by finite differences

$$
\frac{\partial C^{(1 f)}}{\partial t}=\frac{C^{(1 f)}(t, x)-C^{(1 f)}(t-\Delta t, x)}{\Delta t}
$$

The purpose of the numerical analysis is to find the value of $C^{(1 f)}(t, x)$.

Because a numerical approach is used here, another factor can be added by way of refinement of the physical model. The dispersive flux of a species of ion at a concentration gradient is expressed by Fick's law, the second term of $(\mathrm{B} 1), \partial J_{x}^{(1)} / \partial x$, can therefore be rearranged as:

$$
\frac{\partial J_{x}^{(1)}}{\partial x}=\frac{\partial\left(A^{(1)} E C^{(1 f)}-D \frac{\partial C^{(1 f)}}{\partial x}\right)}{\partial x}
$$

With $A^{(1)} E$ the velocity of the ions and $D \partial C^{(1 f)} / \partial x$ the Fick's law element, $D$ being the Fick's law diffusion coefficient.

$$
\frac{\partial J_{x}^{(1)}}{\partial x}=A^{(1)} E \frac{C^{(1 f)}(t-\Delta t, x+\Delta x)-C^{(1 f)}(t-\Delta t, x)}{\Delta x}-D \frac{\partial^{2} C^{(1 f)}}{\partial^{2} x}
$$

To calculate $D \partial^{2} C^{(1 f)} / \partial^{2} x$ a curve fitting method over three points is used, if the three points considered are at $x, x+\Delta x$ and $x+2 \Delta x$, then $D \partial^{2} C^{(1 f)} / \partial^{2} x$ simplifies to $C^{(1 f)}(t-\Delta t, x)-2 C^{(1 f)}(t-\Delta t, x+\Delta x)+C^{(1 f)}(t-\Delta t, x+2 \Delta x), \partial J_{x}^{(1)} / \partial x$ then becomes:

$$
\begin{aligned}
\frac{\partial J_{x}^{(1)}}{\partial x}= & A^{(1)} E \frac{C^{(1 f)}(t-\Delta t, x+\Delta x)-C^{(1 f)}(t-\Delta t, x)}{\Delta x} \\
& -\left(C^{(1 f)}(t-\Delta t, x)-2 C^{(1 f)}(t-\Delta t, x+\Delta x)+C^{(1 f)}(t-\Delta t, x+2 \Delta x)\right)
\end{aligned}
$$

The ion flux in the $y$-direction is described as follows:

$$
\frac{J_{y}^{(1)}}{a}=\frac{P^{(11)} C^{(1 p)}(t-\Delta t, x+\Delta x)+F^{(11)} C^{(1 f)}(t-\Delta t, x+\Delta x)}{a}
$$

Two parameters are still to be chosen: $\Delta x$ and $\Delta t$. Following Carslaw and Jaeger ${ }^{32}$ numerical stability is ensured when the time step is chosen such that $D \Delta t /(\Delta x)^{2} \leq 0.5$. For reasonable spatial resolution $\Delta x=L / 30$.

Knowing the various parameters at $t-\Delta t$ the value of $C^{(1 f)}(t, x+1)$ can be calculated. 
FIGURES AND TABLES

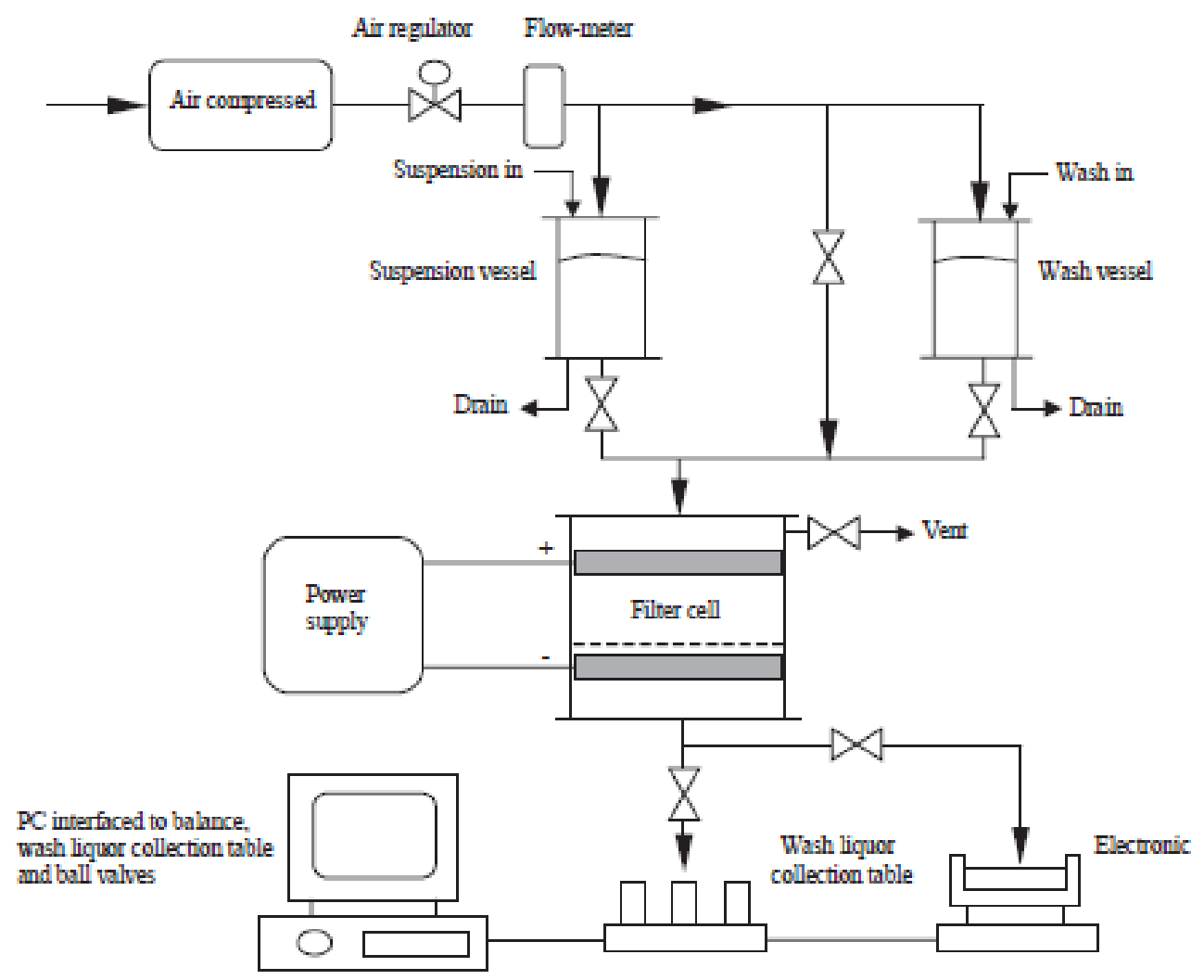

Figure 1: Schematic diagram of the experimental apparatus with the electrode polarity shown in the normal configuration. 


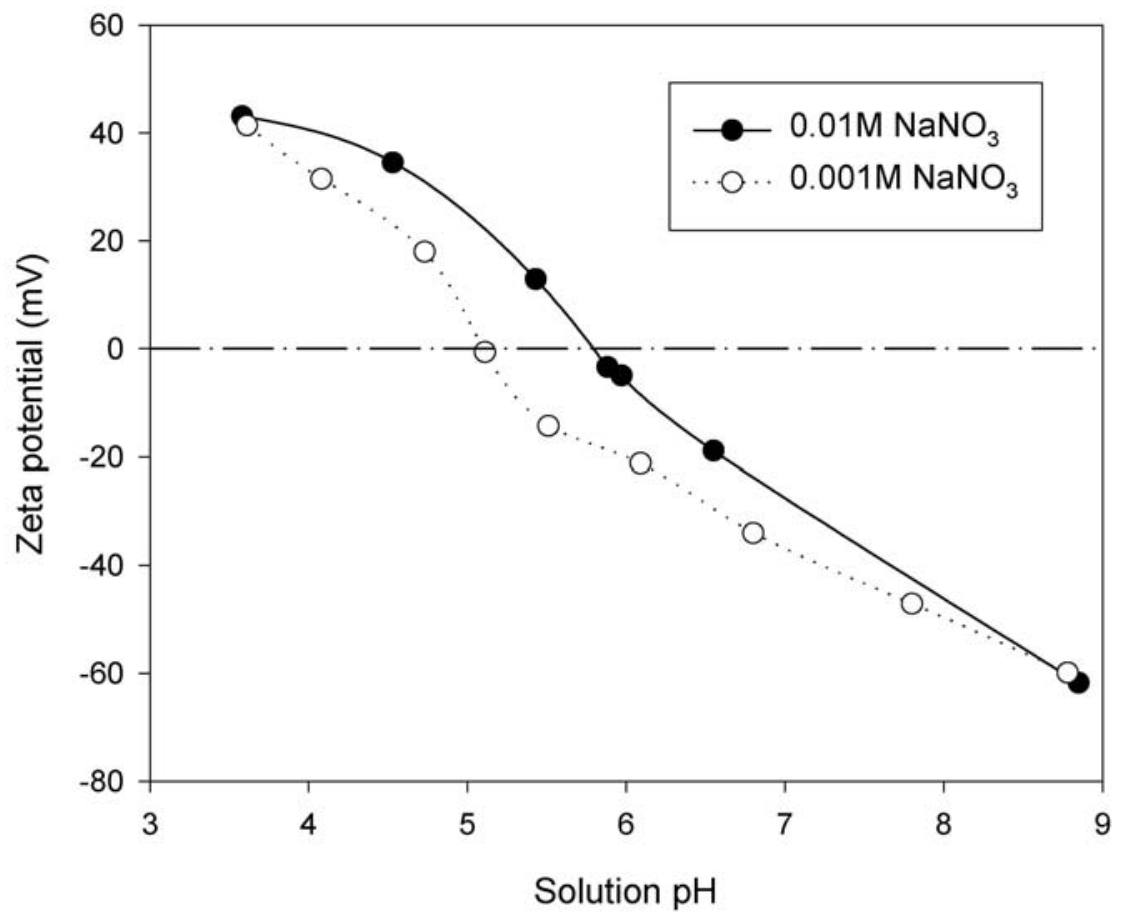

Figure 2: Zeta-potential of $\mathrm{TiO}_{2}$ (rutile) in aqueous solutions, $\mathrm{pH}$ adjusted with either $\mathrm{HNO}_{3}$ or $\mathrm{NaOH}$.

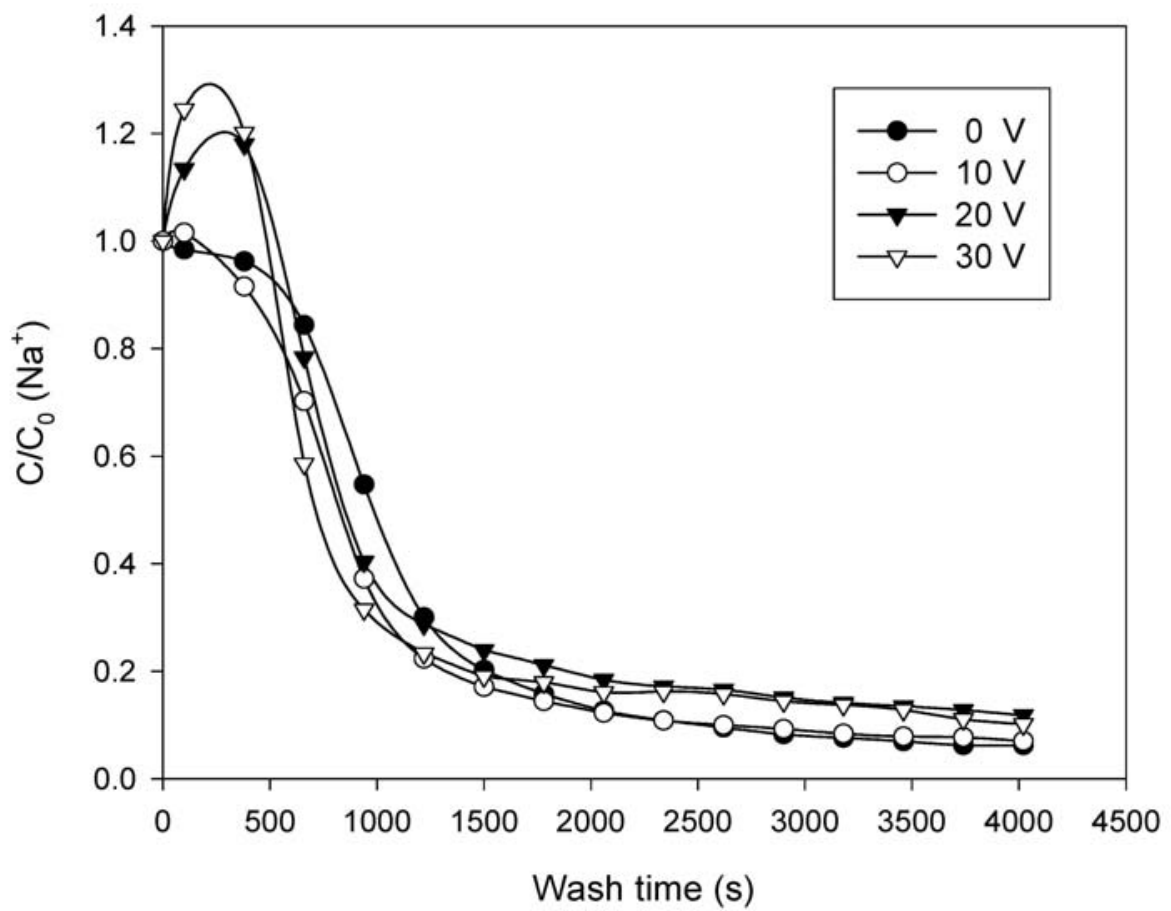

Figure 3: Variations of $C / C_{0}\left(\mathrm{Na}^{+}\right)$and in the wash liquor with time $(t)$ and applied voltage $(\mathrm{pH} 7.8$, $10^{-3} \mathrm{M} \mathrm{NaNO}_{3}, \Delta p_{w}=\Delta p_{f}=400 \mathrm{kPa}$ ). The area under the curve, which corresponds to the quantity of ions removed, is the time for each experiment. However, this can only be seen when a longer period if time is considered. 


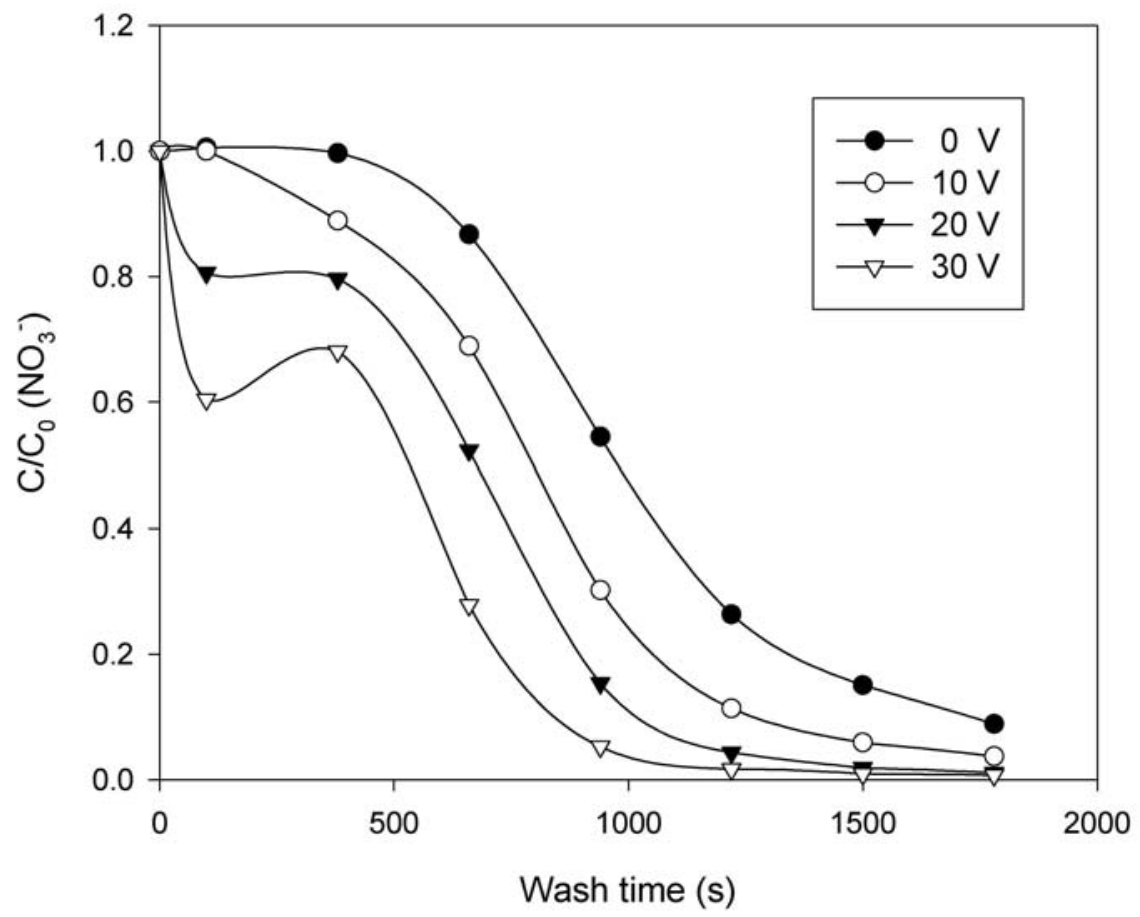

Figure 4: Variations of $\mathrm{C} / \mathrm{C}_{0}\left(\mathrm{NO}_{3}{ }^{-}\right)$in the wash liquor with time $(t)$ and applied voltage $\left(\mathrm{pH} 7.8,10^{-3}\right.$ $\mathrm{M} \mathrm{NaNO}_{3}, \Delta p_{w}=\Delta p_{f}=400 \mathrm{kPa}$ ). The area under the curve, which corresponds to the quantity of ions removed, is the time for each experiment. However, this can only be seen when a longer period if time is considered.

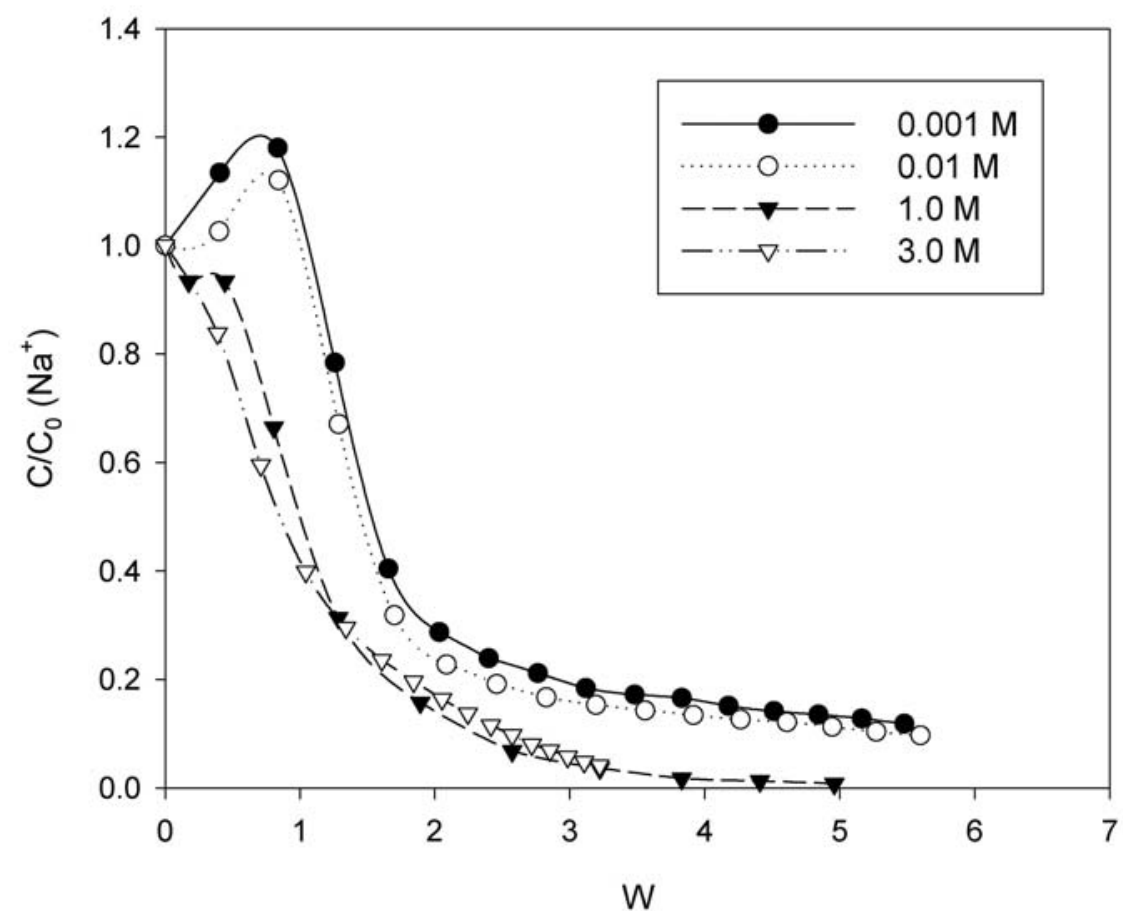

Figure 5: Variations of $C / C_{0}\left(\mathrm{Na}^{+}\right)$in the wash liquor with wash ratio $(W)$ and ionic strength $(10 \mathrm{~mm}$ cake, $\left.\mathrm{pH} 7.4,20 \mathrm{~V}, \Delta p_{w}=\Delta p_{f}=100 \mathrm{kPa}\right)$. 


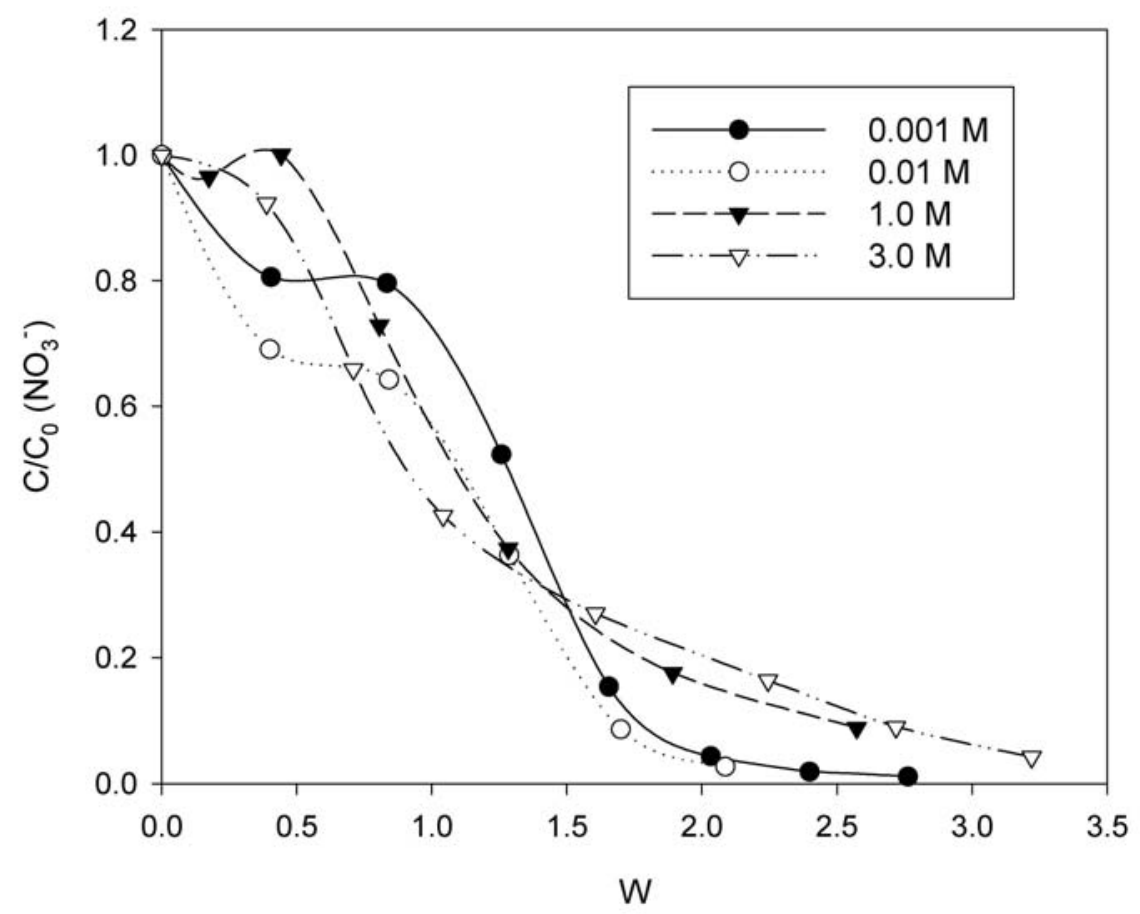

Figure 6: Variations of $C / C_{0}\left(\mathrm{NO}_{3}{ }^{-}\right)$in the wash liquor with wash ratio $(W)$ and ionic strength (10 $\mathrm{mm}$ cake, $\left.\mathrm{pH} 7.4,20 \mathrm{~V}, \Delta p_{w}=\Delta p_{f}=100 \mathrm{kPa}\right)$.

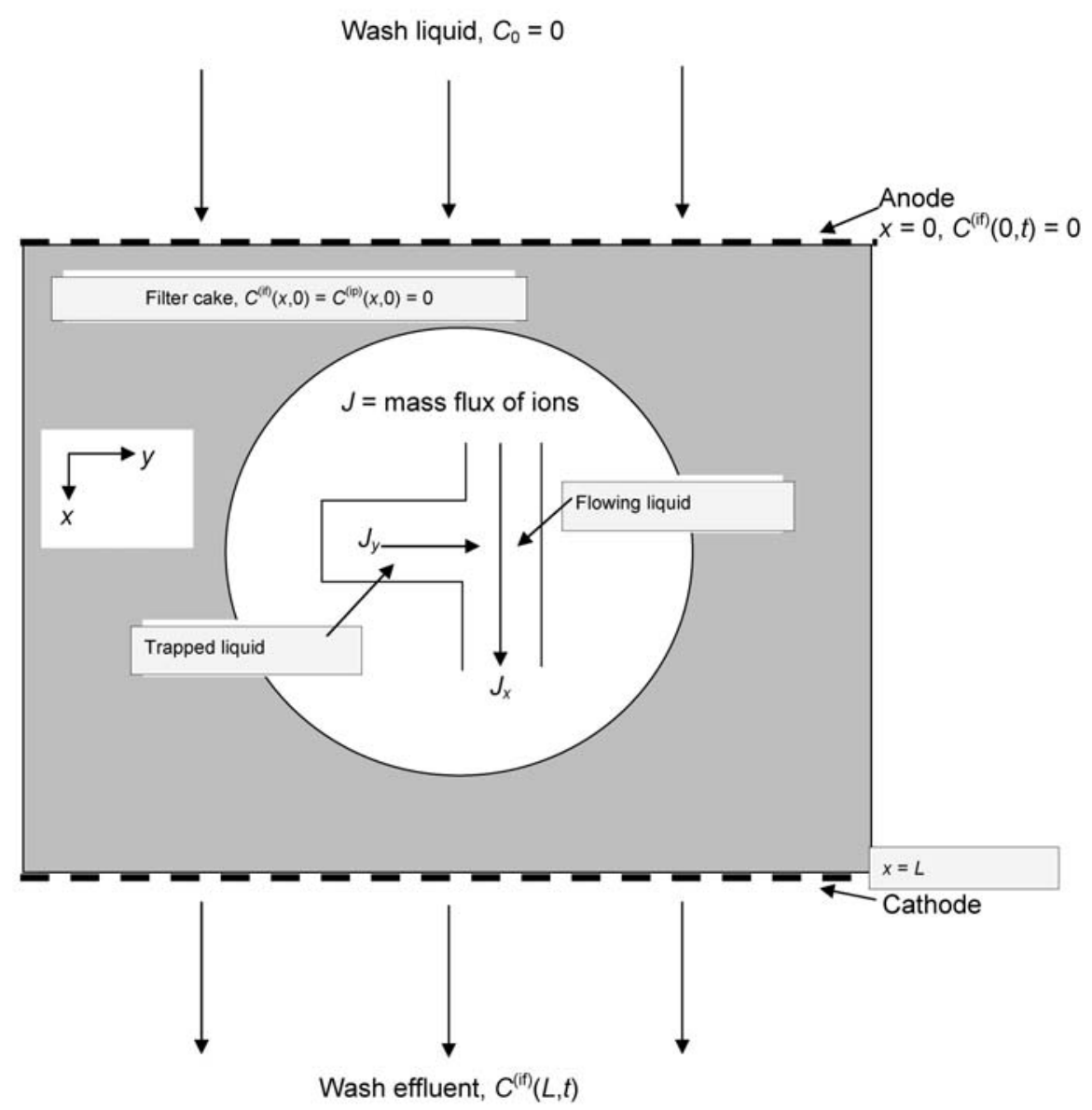

Figure 7: The modelling concept. 


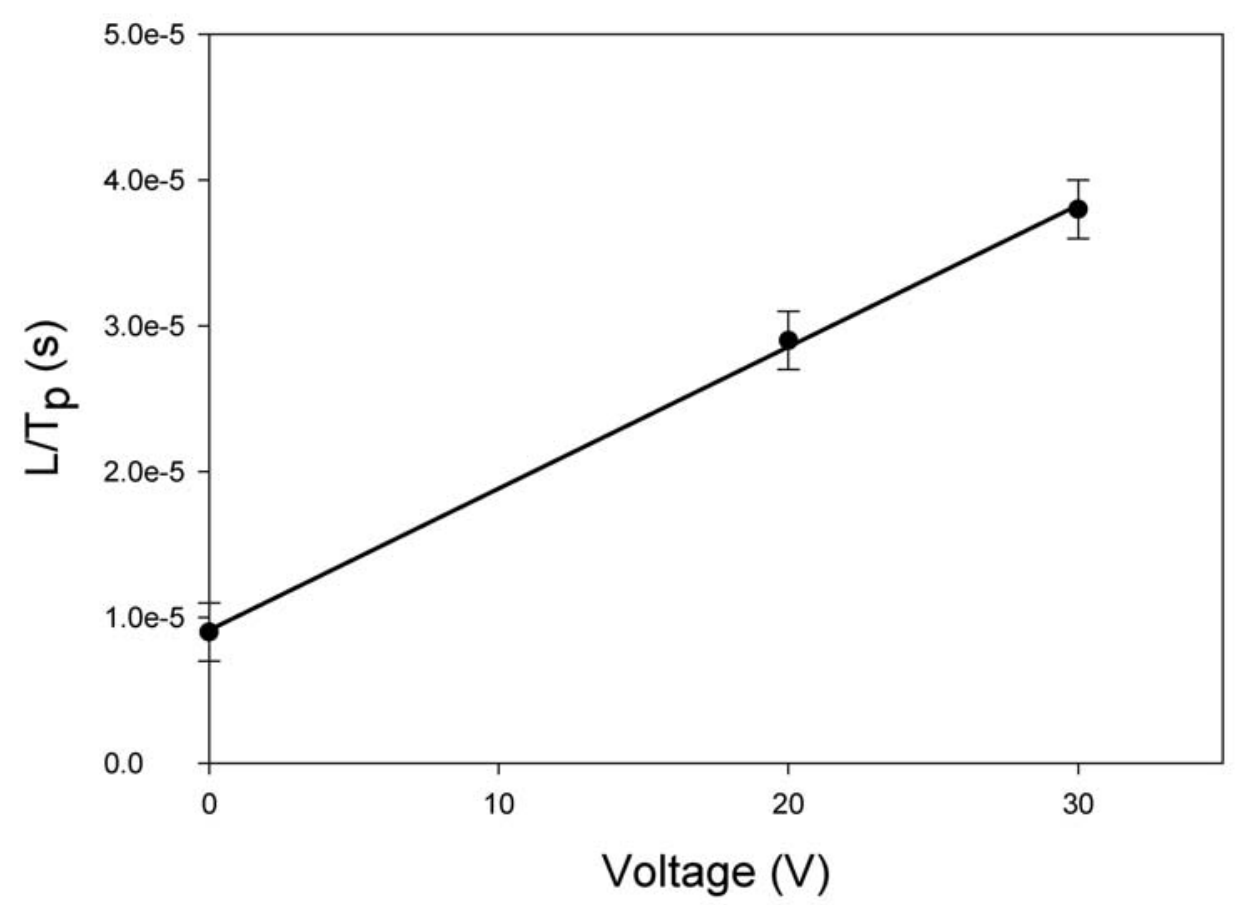

Figure 8: Location of the peak in the washing curve for $\mathrm{pH} 7.8$.

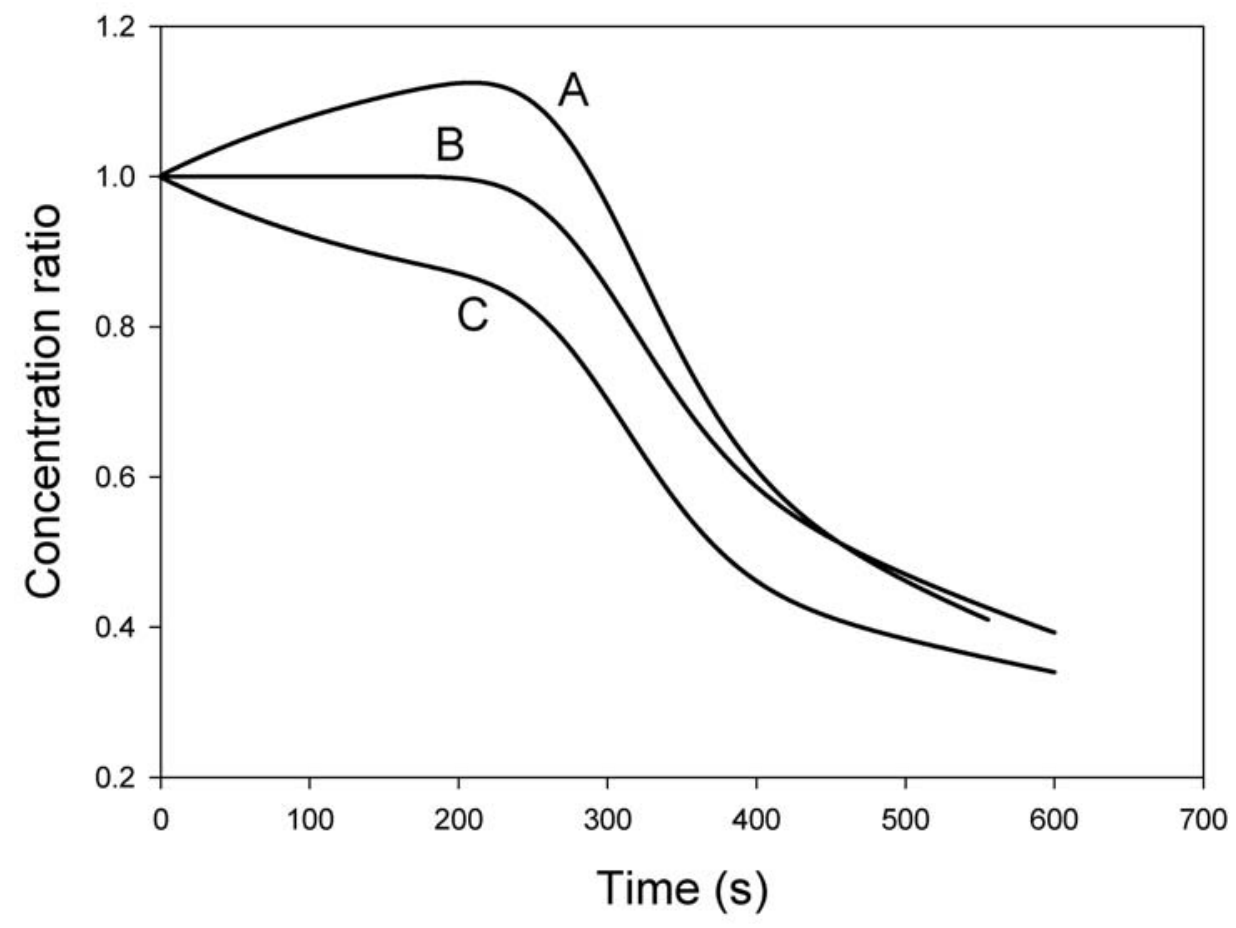

Figure 9: Concentration gradient as calculated by a numerical simulation for three sets of values for $P^{(11)}$ and $F^{(11)} ; \mathrm{A}: P^{(11)} / \mathrm{a}=-0.003 \mathrm{~s}^{-1}, F^{(11)} / \mathrm{a}=0.002 \mathrm{~s}^{-1} ; \mathrm{B}: P^{(11)} / \mathrm{a}=-0.003 \mathrm{~s}^{-1}$, $F^{(11)} / a=0.003 \mathrm{~s}^{-1} ; \mathrm{C}: P^{(11)} / a=-0.002 \mathrm{~s}^{-1}, F^{(11)} / a=0.003 \mathrm{~s}^{-1}$. 


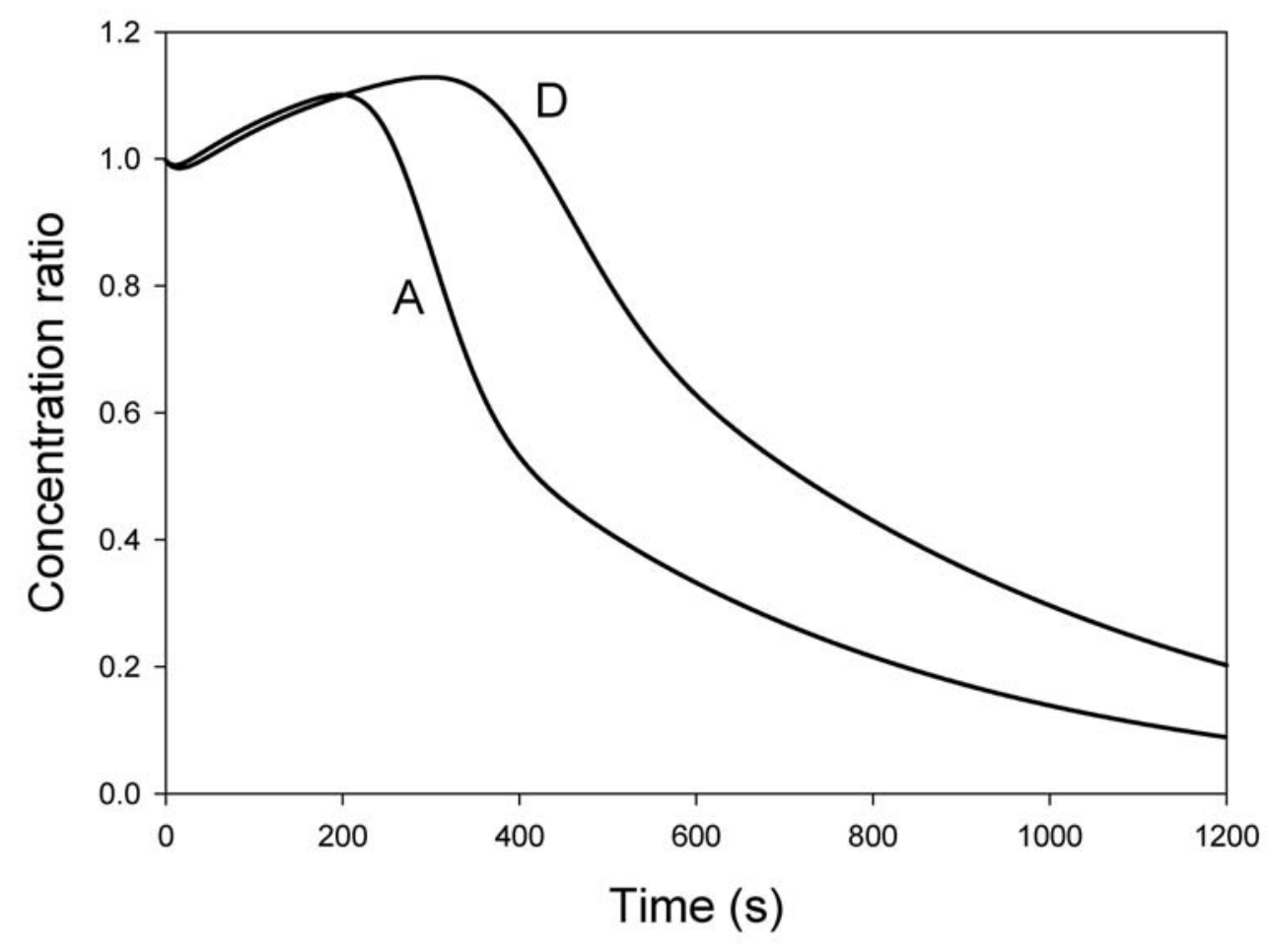

Figure 10: Concentration gradient as calculated by a numerical simulation for two sets of values for the velocity, ionic velocity for $A$ is $3 \times 10^{-5} \mathrm{~m} \mathrm{~s}^{-1}$ and $2 \times 10^{-5}$ for $D . P^{(11)}$ and $F^{(11)}$ are similar for both curves $P^{(11)} / a=-0.003 \mathrm{~s}^{-1}, F^{(11)} / a=0.002 \mathrm{~s}^{-1}$. 


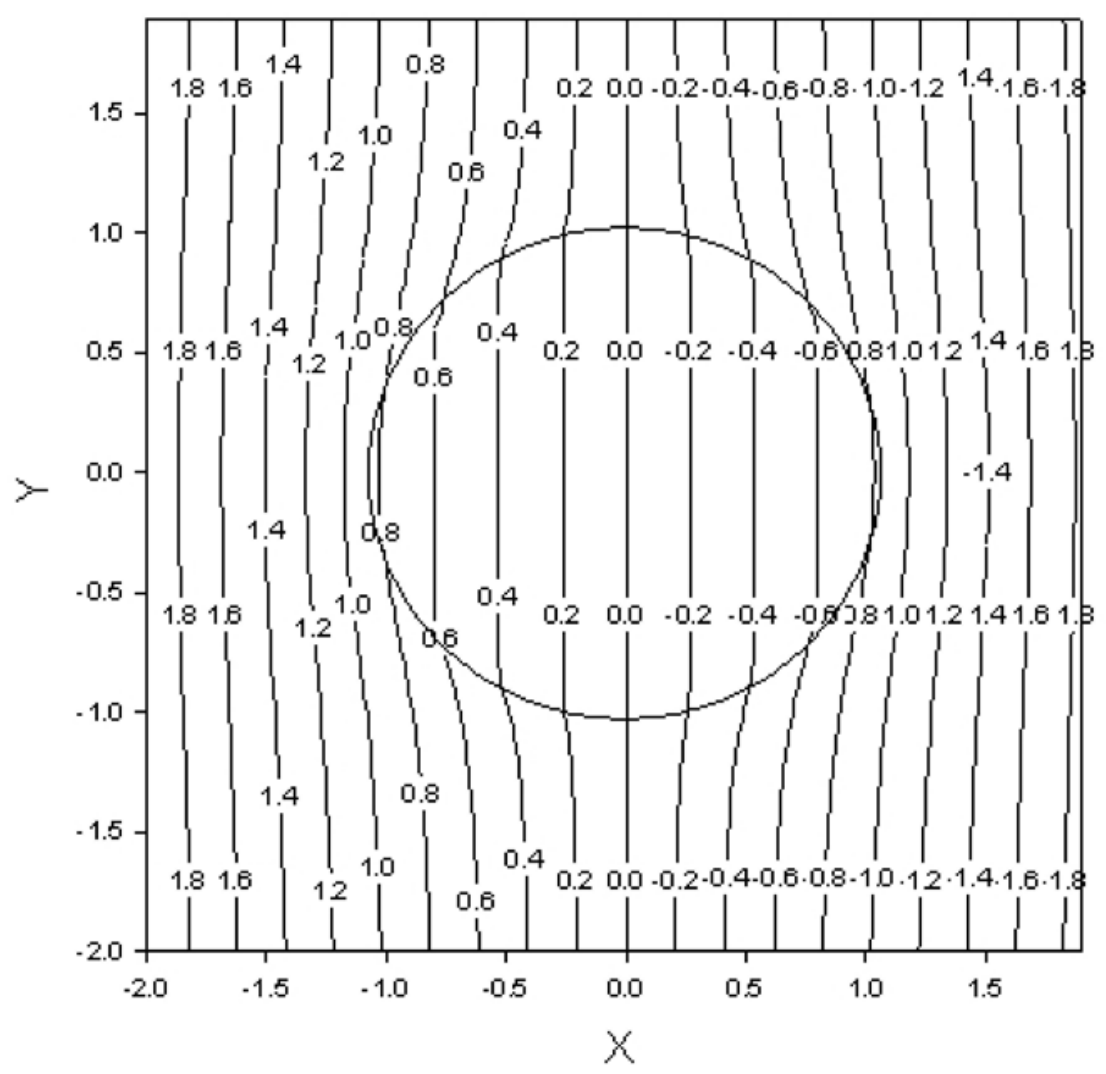

Figure 11: Equipotential lines for a spherical inclusion of different dielectric constant embedded in an otherwise homogeneous medium. The inclusion has diameter equal to 2 and the ratio of the dielectric constant in the inclusion to the dielectric constant in the rest of the medium is 2 .

\begin{tabular}{|l|c|c|c|c|}
\hline & \multicolumn{4}{|c|}{ Velocity $\times 10^{-4} \mathrm{~m} \mathrm{~s}^{-1}$} \\
\hline $\begin{array}{l}\mathrm{pH} \text {, applied pressure, } \\
\text { molarity }\end{array}$ & $\begin{array}{c}\text { Ion migration } \\
\text { velocity } v_{(1)}\end{array}$ & $\begin{array}{c}\text { Electroosmotic } \\
\text { velocity } v_{(2)}\end{array}$ & $\begin{array}{c}\text { Velocity due to } \\
\text { applied pressure } \\
\text { (no field) } v_{(3)}\end{array}$ & $\begin{array}{c}\text { Measured velocity } \\
\text { from peak } v_{(m)}\end{array}$ \\
\hline $7.8,400 \mathrm{kPa}, 10^{-3} \mathrm{M}$ & 1.0 & 0.6 & 0.08 & 0.3 \\
$5.2,100 \mathrm{kPa}, 10^{-3} \mathrm{M}$ & 1.0 & 0.01 & $0.08^{*}$ & $0.18^{*}$ \\
$7.8,400 \mathrm{kPa}, 10^{-2} \mathrm{M}$ & 1.0 & 0.5 & 0.08 & 0.26 \\
\hline
\end{tabular}

${ }^{*}$ Close to the IEP the cake porosity is reduced to an extent dependent on the magnitude of the electric field.

Table 1: Contributions to the overall flux from ion migration, electroosmosis and applied pressure at $20 \mathrm{~V}$ at different $\mathrm{pH}$, applied washing pressure and mother liquor concentration. 